\title{
Prognostic Radiological Factors Affecting Clinical Outcomes of Reverse Shoulder Arthroplasty in the Korean Population
}

\author{
Sung-Min Rhee, MD, Jeong Dong Lee, MD, Yong Bok Park, MD*, \\ Jae Chul Yoo, $\mathrm{MD}^{\dagger}$, Joo Han Oh, MD
}

Department of Orthopedic Surgery, Seoul National University Bundang Hospital, Seoul National University College of Medicine, Seongnam, ${ }^{*}$ Department of Orthopedic Surgery, Soonchunhyang University Bucheon Hospital, Soonchunhyang University School of Medicine, Bucheon, ${ }^{\dagger}$ Department of Orthopedic Surgery, Samsung Medical Center, Sungkyunkwan University School of Medicine, Seoul, Korea

Background: Despite the growing use of reverse shoulder arthroplasty (RSA), it is associated with relatively frequent complications and uncertain clinical outcomes. We investigated radiological factors affecting clinical outcomes of RSA in the Korean population.

Methods: We evaluated physical findings, radiographic findings, visual analog scale scores for pain and satisfaction, and several functional scores in 179 consecutive patients who underwent RSA at two centers between 2008 and 2014.

Results: In 146 included RSAs, pain and forward flexion improved with deltoid lengthening (average, $23.5 \pm 9.1 \mathrm{~mm} ; p=0.039$ ). External rotation decreased with medialization (average, $16.8 \pm 6.0 \mathrm{~mm}, p=0.025$ ), whereas internal rotation showed no correlation with humeral retroversion. Scapular notching $(n=44,30 \%)$ significantly decreased with greater inferior glenosphere overhang (average, $2.94 \pm 3.0 \mathrm{~mm} ; p=0.001$ ), greater prosthesis scapular neck angle (average, $104^{\circ} \pm 10.3^{\circ} ; p=0.001$ ), greater glenoid neck length (average, $9.8 \pm 2.54 \mathrm{~mm} ; p=0.012$ ), lower inferior baseplate tilt angle (average, $105.5^{\circ} \pm 9.2^{\circ} ; p=0.009$ ), and varus humeral neck-shaft angle $(p=0.046)$, and it did not affect ranges of motion and pain, satisfaction, and functional scores. At the final followup, medialization was related to improvement in pain and satisfaction, and inferior glenosphere overhang to functional scores.

Conclusions: Proper amount of deltoid lengthening (mean, $2.3 \mathrm{~cm}$ ) and inferior glenosphere overhang (mean, $2.9 \mathrm{~mm})$ should be chosen for the better outcomes, while the center of rotation should be individualized according to patient characteristics in the Korean population.

Keywords: Rotator cuff tear arthropathy, Reverse shoulder arthroplasty, Prognostic factors, Patient outcomes, Korean population

Received June 14, 2018; Accepted October 14, 2018

Joo Han Oh and Jae Chul Yoo contributed equally to this work as corresponding authors.

Correspondence to: Joo Han Oh, MD

Department of Orthopedic Surgery, Seoul National University Bundang Hospital, Seoul National University College of Medicine, 166 Gumi-ro, Bundanggu, Seongnam 13620, Korea

Tel: +82-31-787-7197, Fax: +82-31-787-4056, E-mail: ohjh1@snu.ac.kr

Co-Correspondence to: Jae Chul Yoo, MD

Department of Orthopedic Surgery, Samsung Medical Center, Sungkyunkwan University School of Medicine, 81 Irwon-ro Gangnam-gu, Seoul 06351, Korea

Tel: +82-2-3410-3501, Fax: +82-2-3410-0061, E-mail: shoulderyoo@gmail.com 
Reverse shoulder arthroplasty (RSA) is known as an effective treatment for cuff tear arthropathy due to promising clinical outcomes, and the indications for RSA are now expanding. ${ }^{1,2)}$ However, the expanded use of RSA has led to an increased number of complications with reported complication rates ranging from 39\% to 59\% including scapular notching. ${ }^{3,4)}$ Complications after RSA often result in poor clinical outcomes, and some of them even necessitate revision surgery. ${ }^{1,2)}$ These high complication rates and variable clinical outcomes have been attributed to positioning of the prosthesis. ${ }^{5,6)}$ Thus, some efforts were proposed to reduce the complication rate and improve the range of motion (ROM), such as inferior glenosphere overhanging, lateralization of the glenosphere, and inferior tilting of the baseplate. However, most of these procedures were developed using cadaver or simulation models, and their effects on clinical outcomes have not been demonstrated ${ }^{5,79)}$ especially in the Asian population.

Shoulder surgery including arthroplasty is becoming more popular in Asia. In addition, the number of RSAs is rapidly increasing owing to the longer life span and broader indications for RSA. Nonetheless, current RSA implants are designed for the United States and western European patients. Eastern Asians usually have a smaller physique, shoulder morphometric features in particular, than the United States and western European populations with the exception of an extended acromial overhang. ${ }^{10)}$ These anatomical differences might result in different clinical outcomes and complications of RSA in the Asian population, ${ }^{11)}$ yet there have been no previous studies regarding factors that might affect the results of RSA in this patient group. Therefore, the purpose of the current study was to determine the prognostic radiological factors affecting the complication rate and clinical outcomes of RSA for cuff tear arthropathy in the Korean population.

\section{METHODS}

We evaluated 197 consecutive RSA cases from two hospitals (Seoul National University Bundang Hospital [SNUBH] and Samsung Medical Center [SMC]) between 2008 and 2014. We conducted this study in compliance with the principles of the Declaration of Helsinki. The protocol of this study was reviewed and approved by the Institutional Review Boards of the Seoul National University Bundang Hospital (IRB No. SNUBH B-1702/384-106) and Samsung Medical Center (IRB No. SMC 2018-12-074001). The informed consent was waived. All procedures were performed by two surgeons (JHO and JCY) in each hospital. Patients in need of revision arthroplasty and with rheumatoid arthritis, consequences of shoulder sepsis, and sequelae of proximal humerus fracture were excluded because these pathologies are known as independent predictors of poor prognosis after RSA. ${ }^{12)}$ RSA patients with cuff tear arthropathy and a massive irreparable cuff tear with a mean follow-up of 20.6 months (range, 12 to 64 months) were included. Finally, 146 consecutive RSAs ( 75 cases from SNUBH and 71 cases from SMC) in 138 patients were analyzed. The following prostheses were implanted: Trabecular Metal (Zimmer, Warsaw, IN, USA; 21 cases), Aequalis System (Tornier, Montbonnot-Saint-Martin, France; 59 cases), Biomet Comprehensive (Biomet, Warsaw, IN, USA; 53 cases), and Reverse System (DJO, Vista, CA, USA; 13 cases). Radiological evaluation was done to assess prognostic factors affecting outcomes of the surgery.

\section{Surgical Procedures}

All patients underwent surgery in the beach chair position using a deltopectoral approach. The humeral stem was inserted with $10^{\circ}$ to $40^{\circ}$ retroversion (SNUBH: following patient's native retroversion using a preoperative torsional computed tomography [CT] scan; SMC: $20^{\circ}$ retroversion only). A cemented stem was used in seven patients because of severe osteoporosis. We attempted to place the inferior rim of the baseplate onto the inferior rim of the bony glenoid with a slight inferior tilt of $10^{\circ}$ to $20^{\circ}{ }^{13)} \mathrm{Al}$ though four different implants were used, all inserted glenospheres were $36 \mathrm{~mm}$ in diameter for avoiding unnecessary effect of the size of glenosphere on outcomes; a large size glenosphere can reduce the rate of scapular notching. After insertion of a trial humeral stem and spacer, proper tension was checked. In the SNUBH, we checked the tension without full reduction of the humeral stem to the glenosphere. If the lateral half of the glenosphere could be seen while pulling the arm in $40^{\circ}$ of external rotation, we assumed that proper tension had been achieved. In the SMC, proper tension was subjectively checked by doing $1 \mathrm{~mm}$ push-pull test: lengthening the arm approximately $1.5 \mathrm{~cm}$, about $1-2$ finger width between the greater tubercle and the acromion, and stimulating snapfit reduction. When the tension was considered proper, three bone tunnels were made at the lesser tuberosity and 2-0 Ethibond sutures (Ethicon, Somerville, NJ, USA) were passed through the bone tunnels. After reduction of the real glenoid and humeral component, the tension of the conjoined tendon and deltoid contour was checked for proper tensioning. If possible, the tenotomized subscapularis tendon was repaired using the transosseous technique with the penetrated Ethibond sutures, followed by biceps tenodesis. 
After surgery, patients were immobilized for 4 weeks. From the day of surgery, patients started passive range of motion (ROM) exercises involving passive forward flexion and external rotation in a tolerable range. Active assisted exercises were started at 4 weeks postoperatively and muscle strengthening exercises were introduced at 2 months after the surgery.

\section{Clinical Assessments}

Visual analog scale (VAS) for pain and patient satisfaction (from 0 to 10 ) was used to evaluate the subjective outcomes. Physical examinations were conducted by two trained shoulder surgeons. The active ROM was measured before surgery, at 3 months, 6 months, and 1 year after surgery, and then annually. A dynamometer was used to measure the isometric muscle strength in abduction. The functional outcome was evaluated using the American Shoulder and Elbow Surgeons (ASES) score, Simple Shoulder Test (SST), Constant score, and Korean Shoulder Score (KSS) at 6 months and 1 year after surgery and then annually.

\section{Radiological Assessments}

Radiographic images were obtained: true anteroposterior view of the glenohumeral joint in the neutral position, lateral view, and axillary view on $\mathrm{X}$ ray; humeral retroversion was assessed using torsional CT. Differences between preoperative and postoperative radiographic values of medialization of the center of rotation (COR) and lengthening of the deltoid were calculated for all patients. ${ }^{14)}$ The scapular neck length was evaluated preoperatively using a true anteroposterior view of the glenohumeral joint in the neutral position, ${ }^{15)}$ and glenosphere overhang, inferior baseplate tilt angle (IBPT), and prosthesis scapular neck angle (PSNA) were measured postoperatively. ${ }^{14,16)}$ Postoperative radiographs taken 4 weeks after surgery were used to measure deltoid lengthening, medialization of the COR, inferior glenosphere overhang, IBPT, and PSNA. Scapular notching was graded according to the classification of Sirveaux et al. ${ }^{17)}$ using radiographs from the last follow-up (Fig. 1).

\section{Statistical Analysis}

All statistical analyses were performed using SPSS ver. 12.0 (SPSS Inc., Chicago, IL, USA). Pearson correlation analysis was used to investigate correlations between radiographic findings and clinical outcomes including ROM, VAS for pain and satisfaction, and functional scores. The paired $t$-test and Fisher exact test were used to evaluate implant design, clinical outcomes, and the incidence of scapular notching. Logistic regression analysis was used to determine factors affecting the incidence of notching. A $p$-value of $<0.05$ was considered to indicate a statistically significant difference.

\section{RESULTS}

Total 146 RSAs in 138 patients with 26 males and 112 females were analyzed. Their mean age was $71 \pm 5.7$ years (range, 57 to 86 years). The dominant arm was affected in 90 patients (65\%), and both shoulders were operated in eight patients (6\%). Among 146 RSAs, 24 cases had intact subscapularis, 59 cases had high grade partial tear of the subscapularis. The remaining 63 cases had a full thickness tear of the subscapularis. One hundred twenty cases had subscapularis repair after insertion of implants. Twentysix cases did not have subscapularis repair due to their full thickness tear of the subscapularis or excessive ten-
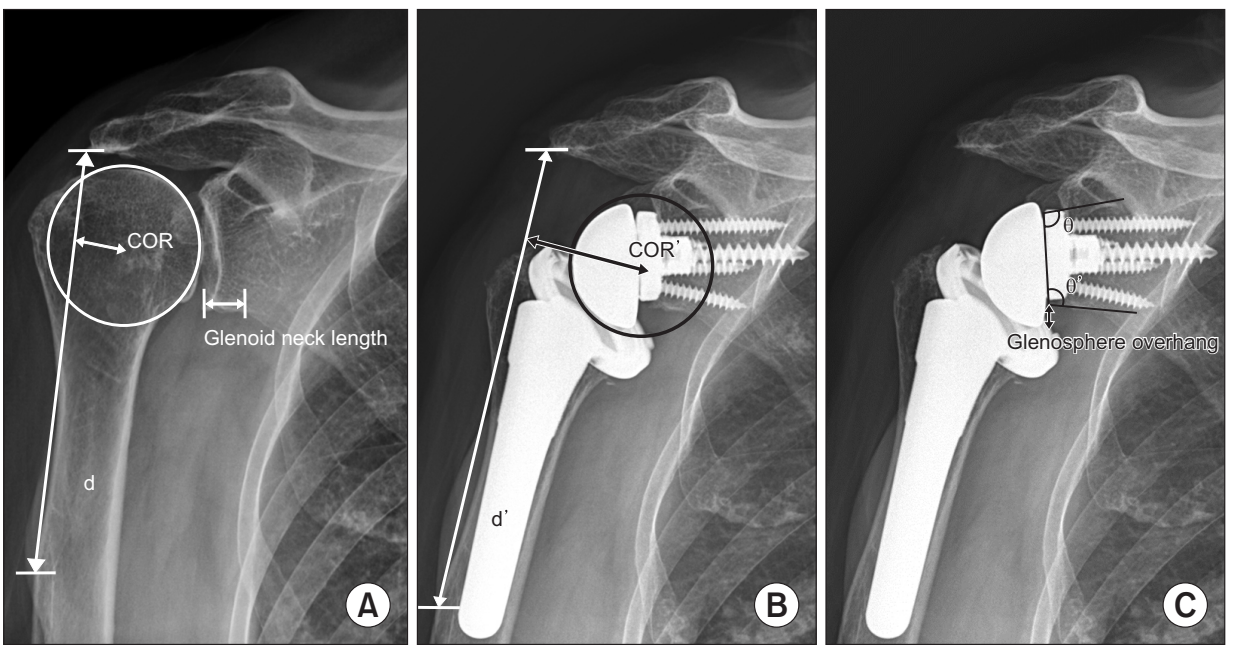

Fig. 1. (A) Preoperatively, the center of rotation (COR), deltoid length $(d)$, and glenoid neck length were measured. (B) Postoperative center of rotation (COR') and deltoid length ( $d$ ') were measured at 4 weeks after surgery. (C) Glenosphere overhang, inferior baseplate tilt angle $(\theta)$, and prosthesis scapular neck angle $\left(\theta^{\prime}\right)$ were also measured. 
sion on the bone tunnels located at the lesser tuberosity. There were no significant differences between two centers in patients' age and sex (Table 1). Similarly, no significant differences were found in ROM, VAS for pain and satisfaction, and functional scores between two hospitals.

After a mean follow-up period of 20.6 months (range, 12 to 64 months), subjective improvement in satisfaction VAS by 6 points was reported in 130 RSAs (89.0\%). The pain VAS was significantly improved postoperatively (preoperative, 6.8; range, 1 to 10; postoperative, 2.5; range, 0 to $7 ; p<0.001$ ). All functional scores were also improved postoperatively: ASES, from 35.9 (range, 7 to 62 ) to 67.3 (range, 22 to 93); SST, from 2.6 (range, 0 to 7 ) to 5.9 (range, 1 to 10); KSS, from 37.9 (range, 9 to 81) to 69.3 (range, 27 to 100); Constant score, from 26.0 (range, 0 to 73 ) to 53.9 (range, 23 to 90). Active forward flexion was significantly increased from $96.4^{\circ}$ (range, $0^{\circ}$ to $155^{\circ}$ ) to $138.4^{\circ}$ (range, $40^{\circ}$ to $\left.175^{\circ}\right)$ postoperatively $(p<0.001)$ and external rotation at the side was also significantly improved from $30.6^{\circ}$ (range, -20 to 75 ) to $48.9^{\circ}$ (range, $10^{\circ}$ to $80^{\circ}$ ) postoperatively $(p<0.001)$. However, there was no significant improvement in internal rotation at the back: it changed from 12.5 (sacrum to T4) preoperatively to 11.2 (sacrum to T4) postoperatively at the vertebral level $(p=0.473)$ (Table 1). The range of forward flexion was decreased in 15 patients from $127.6^{\circ}$ (range, $90^{\circ}$ to $170^{\circ}$ ) at 1 year after surgery to $94.3^{\circ}$ (range, $60^{\circ}$ to $120^{\circ}$ ) at the final follow-up $(p=0.048)$. External rotation and internal rotation at the back were maintained: external rotation, from $29.3^{\circ}$ (range, $10^{\circ}$ to $30^{\circ}$ ) to $25.7^{\circ}$ (range, $10^{\circ}$ to $30^{\circ}$ ); internal rotation at the back, from L1 (range, sacrum to T4) to L1 (range, L5 to T7); $p=0.671$ and $p=0.887$, respectively. The mean final follow-up period was 35.8 months (range, 32 to 123 months).

There were no cases with instability, implant failure, and acromial stress fracture. However, superficial infection occurred in three patients after RSA and it was managed with intravenous antibiotics only. Seven patients reported transient neurologic complications, including transient axillary nerve neuropathy $(\mathrm{n}=2)$ and tingling sensation of the upper arm, which recovered by 6 months postoperatively. The average of deltoid lengthening after the surgery in those patients was $24.1 \pm 9.8 \mathrm{~mm}$.

Considering the correlation between radiographic parameters and postoperative ROM, greater deltoid lengthening (average, $23.5 \pm 9.1 \mathrm{~mm}$; range, 5.1 to 43.7 ; $p=0.04$ ) and greater medialization of the COR (average, $16.8 \pm 6.0 \mathrm{~mm}$; range, 5.3 to 34.3 ; $p=0.04$ ) were significantly correlated with increased postoperative active forward flexion (Fig. 2). In contrast, greater medialization of the COR significantly decreased external rotation at the side ( $p=0.03)$ (Fig. 3). Internal rotation at the back

Table 1. Demographics and Clinical Characteristics of the Patients at Two Centers

\begin{tabular}{|c|c|c|c|c|}
\hline Variable & SNUBH $(n=75)$ & $\operatorname{SMC}(n=71)$ & Total $(n=146)$ & $p$-value \\
\hline Age (yr) & $71.1 \pm 5.7$ & $71.8 \pm 6.5$ & $71.4 \pm 5.7$ & 0.534 \\
\hline Sex (male:female) & $13: 62$ & $15: 56$ & 28:118 & 0.756 \\
\hline$F F\left({ }^{\circ}\right)$ & $139.0 \pm 27.7$ & $137.7 \pm 26.5$ & $138.4 \pm 27.1$ & 0.635 \\
\hline ER at side $\left(^{\circ}\right)$ & $50.7 \pm 19.3$ & $47.9 \pm 19.7$ & $48.9 \pm 19.5$ & 0.132 \\
\hline IR at back & $10.8 \pm 4.0$ & $11.6 \pm 3.6$ & $11.2 \pm 3.8$ & 0.189 \\
\hline Notching & $24(32)$ & $20(28)$ & $44(30)$ & 0.531 \\
\hline Pain (VAS) & $2.2 \pm 1.6$ & $2.8 \pm 1.7$ & $2.5 \pm 1.7$ & 0.166 \\
\hline Satisfaction (VAS) & $7.3 \pm 2.4$ & $6.7 \pm 2.1$ & $7.0 \pm 2.2$ & 0.137 \\
\hline ASES score & $69.4 \pm 16.7$ & $65.0 \pm 18.8$ & $67.3 \pm 17.8$ & 0.203 \\
\hline SST & $6.2 \pm 3.9$ & $5.5 \pm 2.3$ & $5.9 \pm 3.3$ & 0.571 \\
\hline KSS & $72.5 \pm 18.7$ & $66.3 \pm 13.8$ & $69.3 \pm 16.5$ & 0.109 \\
\hline Constant score & $56.8 \pm 18.4$ & $51.0 \pm 13.0$ & $53.9 \pm 16.1$ & 0.124 \\
\hline
\end{tabular}

Values are presented as mean \pm standard deviation or number (\%).

Internal rotation was measured as the level of vertebral spinous process that could be reached with the patient's thumb.

SNUBH: Seoul National University Bundang Hospital, SMC: Samsung Medical Center, FF: forward flexion, ER: external rotation, IR: internal rotation, VAS: visual analog scale, ASES: American Shoulder and Elbow Surgeons, SST: Simple Shoulder Test, KSS: Korean Shoulder Score. 


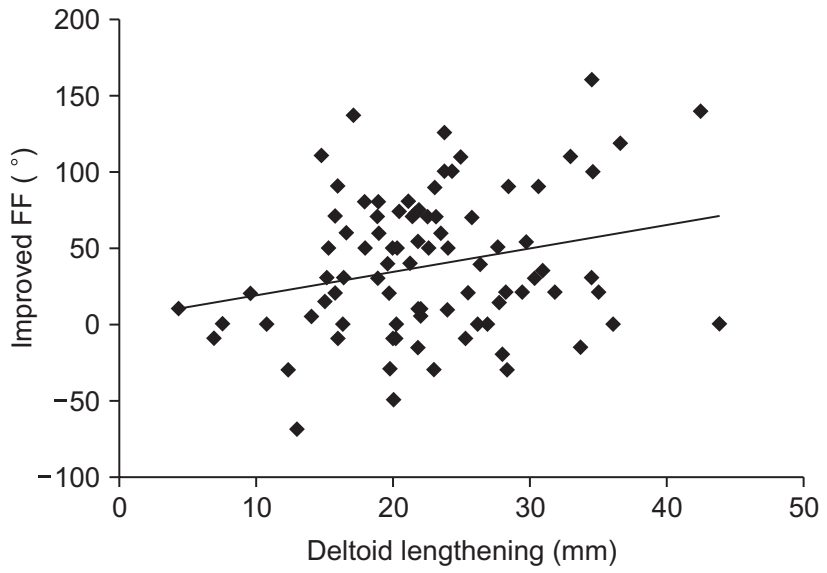

Fig. 2. Greater deltoid lengthening (average, $23.5 \pm 9.1 \mathrm{~mm} ; p=0.04$ ) was significantly correlated with increased active forward flexion (FF) postoperatively.

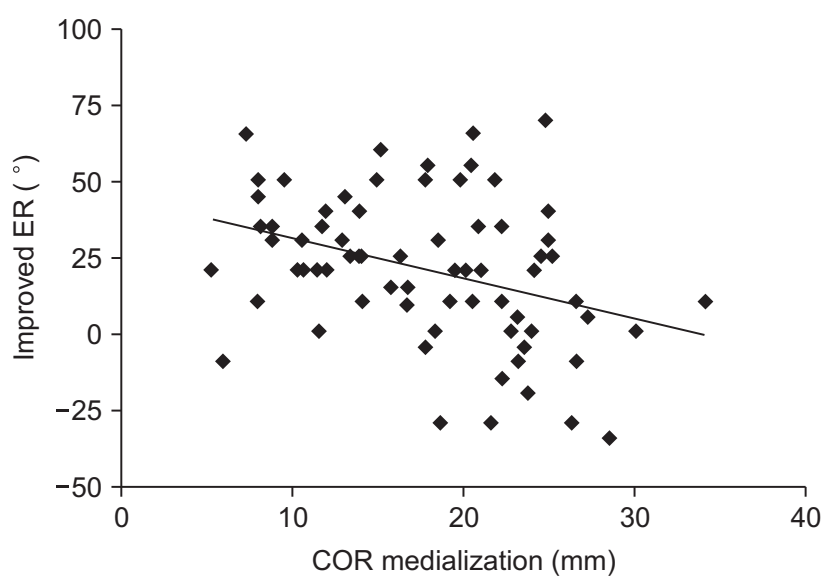

Fig. 3. Greater medialization of the center of rotation (COR; average, 16.8 $\pm 6.0 \mathrm{~mm} ; p=0.03$ ) was significantly associated with decreased external rotation (ER) at the side.

showed no significant correlation with any of radiographic parameters, including humeral retroversion angle.

Scapular notching was found in 44 RSAs $(30 \%, 37$ grade 1 and 7 grade 2) at the final follow-up. There was no significant difference in the prevalence of scapular notching between hospitals. Regardless of scapular notching, differences in clinical outcomes were insignificant, and there were no correlations between scapular notching and pain, satisfaction, active ROM, and functional scores.

However, some radiographic parameters were significantly related to the incidence of scapular notching. For the humeral component, the increased neck-shaft angle (valgus inclination of $155^{\circ}$ ) was significantly related to the increased prevalence of notching compared to the stem varus inclination angle of $135^{\circ}-145^{\circ}$. For the glenoid com-

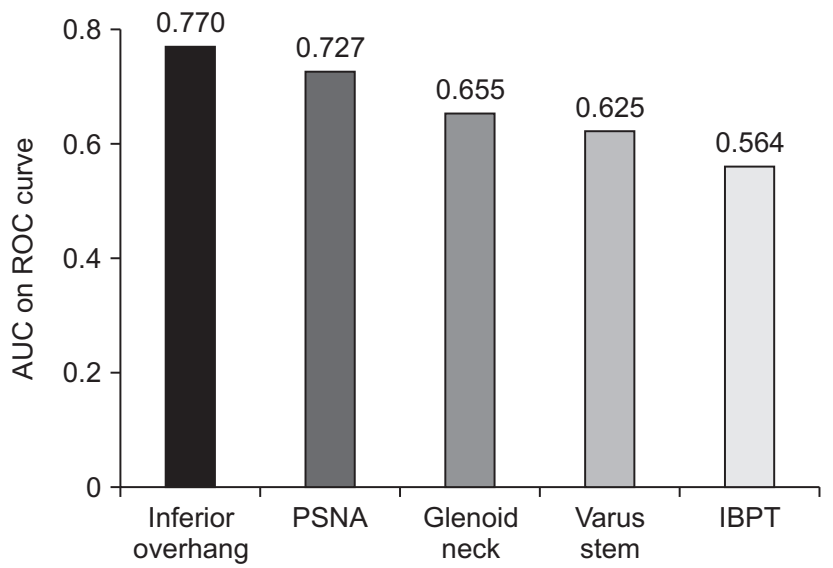

Fig. 4. Receiver operating characteristic (ROC) curve analysis indicates that inferior glenosphere overhang (area under curve, 0.77; cutoff value, $2.645 \mathrm{~mm}$ ) is the most important factor in preventing notching. AUC: area under curve, PSNA: prosthesis scapular neck angle, IBPT: inferior baseplate tilt angle.

ponent, inferior glenosphere overhang $(2.94 \pm 3.0 \mathrm{~mm}$; range, -1.4 to $8.5 \mathrm{~mm})$, decreased PSNA $\left(104^{\circ} \pm 10.3^{\circ}\right.$; range, $90.4^{\circ}$ to $\left.122.2^{\circ}\right)$, increased glenoid neck length $(9.8$ $\pm 2.54 \mathrm{~mm}$; range, 4.4 to $14.9 \mathrm{~mm}$ ), and increased IBPT were significantly related to the decreased prevalence of scapular notching. The receiver operating characteristic (ROC) curve analysis indicated that inferior glenosphere overhang was the most important factor in preventing notching (area under the curve [AUC], 0.77) (Fig. 4).

Some subjective outcomes were significantly affected by certain radiographic parameters as well. Greater medialization of the COR was associated with significant improvement in the postoperative VAS for pain $(p=0.018)$ and satisfaction $(p=0.021)$; however, other functional scores were not related with COR medialization. Inferior glenosphere overhang significantly affected all measured functional scores. The ASES ( $p=0.037)$, SST $(p=0.018)$, Constant score $(p=0.022)$, and KSS $(p=0.035)$ were all significantly improved postoperatively with more inferior glenosphere overhanging. Other radiographic measurements, COR, IBPT, and PSNA did not correlate with postoperative function (Table 2).

\section{DISCUSSION}

The mid-term functional outcomes of RSA in the Korean population appear to be satisfactory, with some of the radiographic measurements significantly affecting the clinical outcomes. Forward flexion increased with deltoid lengthening and medialization of the COR and external rotation decreased with medialization, whereas internal 
Rhee et al. Outcomes of Reverse Total Shoulder Arthroplasty in Korean Population

Clinics in Orthopedic Surgery • Vol. 11, No. 1, 2019•www.ecios.org

Table 2. Clinical Outcomes According to Radiographic Measurements

\begin{tabular}{|c|c|c|c|c|c|c|c|c|c|}
\hline \multirow{2}{*}{ Variable } & \multirow{2}{*}{ Pain } & \multirow{2}{*}{ Satisfaction } & \multicolumn{3}{|c|}{ ROM } & \multicolumn{4}{|c|}{ Functional score } \\
\hline & & & $\mathrm{FF}$ & ER & IR & ASES score & SST & KSS & Constant \\
\hline Notching & 0.411 & 0.129 & 0.273 & 0.582 & 0.172 & 0.972 & 0.171 & 0.637 & 0.804 \\
\hline COR medialization & $0.007^{*}$ & $0.011^{*}$ & 0.796 & $0.023^{*}$ & 0.280 & 0.092 & 0.127 & 0.070 & 0.054 \\
\hline Glenoid neck length & 0.329 & 0.536 & 0.362 & 0.978 & 0.628 & 0.764 & 0.619 & 0.397 & 0.629 \\
\hline Deltoid lengthening & 0.076 & 0.180 & $0.048^{*}$ & 0.626 & 0.196 & 0.835 & 0.661 & 0.440 & 0.840 \\
\hline IBPT & 0.159 & 0.973 & 0.278 & 0.130 & 0.366 & 0.815 & 0.354 & 0.594 & 0.436 \\
\hline PSNA & 0.453 & 0.483 & 0.634 & 0.657 & 0.594 & 0.198 & 0.185 & 0.150 & 0.285 \\
\hline Glenoid overhang & 0.432 & 0.876 & 0.120 & 0.540 & 0.727 & $0.043^{*}$ & $0.016^{*}$ & $0.020^{*}$ & $0.022^{*}$ \\
\hline Humeral stem angle & 0.350 & 0.452 & 0.134 & 0.172 & 0.386 & 0.364 & 0.147 & 0.222 & 0.454 \\
\hline
\end{tabular}

ROM: range of motion, FF: forward flexion, ER: external rotation, IR: internal rotation, ASES: American Shoulder and Elbow Surgeons score, SST: Simple Shoulder Test, KSS: Korean Shoulder Score, COR: center of rotation, IBPT: inferior baseplate tilt angle, PSNA: prosthesis scapular neck angle.

${ }^{*}$ Significant difference $(p<0.05)$ in clinical outcomes.

rotation showed no correlation with humeral retroversion. The ROC curve analysis indicated that inferior glenosphere overhang was the most important factor for preventing scapular notching in this Korean population.

Few clinical studies have focused on determining the optimal extent of deltoid lengthening that would allow satisfactory forward flexion and decrease the rate of complications including prosthetic instability, neurologic complication, and acromial stress fracture. ${ }^{18-20)}$ In the current study, active forward flexion significantly increased postoperatively $\left(90.1^{\circ}\right.$ to $\left.138.4^{\circ}\right)$ and as expected, it was significantly associated with deltoid lengthening (average, $23.5 \pm 9.1 \mathrm{~mm} ; p=0.039$ ). While the current results are similar to previous studies which recommended 2 to $3 \mathrm{~cm}$ lengthening of the arm, ${ }^{14,21)}$ this is the first study involving a large group of patients, especially in the Korean population.

It is still a matter of debate whether medialization or lateralization of the COR yields better clinical outcomes and reduces the complication rate. In the current study, greater medialization had both advantages and disadvantages. One of the noted advantages was that active forward flexion significantly increased $(p=0.046)$ with increasing medialization (average, $16.8 \pm 6.0 \mathrm{~mm}$ ). This is in contradiction to the study of a smaller patient group from a single center which showed no correlation between forward flexion and medialization. ${ }^{14)}$ Furthermore, postoperative VAS for pain $(p=0.018)$ and satisfaction $(p=0.021)$ significantly increased with greater medialization. The results of this study were similar to other studies showing the advantages of greater medialization. Recently, Hoenecke et al. ${ }^{9)}$ reported that greater medialization resulted in decreased glenohumeral joint reaction force and decreased deltoid muscle force in abduction based on scapulohumeral models reconstructed from CT scans. Similarly, $10 \mathrm{~mm}$ lateralization of the glenosphere significantly increased shear displacement of the baseplate compared to medial position in a biomechanical study. ${ }^{22)}$ These previous studies support the current data where more medialization resulted in improved forward flexion, pain and, satisfaction postoperatively. On the contrary, regarding disadvantages of medialization, greater medialization was significantly associated with worse external rotation at the side in the current study. Previous studies demonstrated improved external rotation after lateralization, which means less medialization of the COR. ${ }^{18,23)}$ Frankle et al ${ }^{18)}$ reported significantly increased active external rotation with less medialized RSA in a series of 60 patients with a minimum follow-up of 2 years. Cuff et al. ${ }^{23)}$ also reported improved active external rotation in 96 patients with less medialized RSA. Better external rotation seems to be achieved by improving the tension of the remaining rotator cuff muscles and posterior deltoid muscle recruitment. ${ }^{24)}$ All in all, medialization of the COR during RSA would help to improve forward flexion and reverse pseudoparalysis; however, this should be done with care in patients who have less preoperative range of external rotation.

Scapular notching is the most common complication of RSA and has been suggested as a cause of glenoid loosening. ${ }^{8,17,25)}$ According to earlier studies, the overall incidence of scapular notching ranges from $44 \%$ to $96 \%{ }^{8,12,25,26)}$ While Levigne et al. ${ }^{8}$ found no correlation 
of scapular notching with pain scores and clinical findings, Sirveaux et al. ${ }^{17)}$ showed a negative effect of scapular notching on clinical outcome, at least in terms of the Constant score. In the present mid-term follow-up study with the Korean population, the occurrence of scapular notching did not significantly affect pain, satisfaction, active range of motion, and functional scores. However, scapular notching may lead to prosthesis instability, unexplained pain, and loosening of components in the longer-term. Therefore, surgeons should strive to avoid notching until more definitive conclusions can be drawn.

Several authors have recommended inferior placement of the glenosphere relative to the glenoid face to reduce the risk of notching. ${ }^{6,8)}$ In addition, in patients with laterally offset glenosphere, the incidence of scapular notching significantly decreased. ${ }^{18,23)}$ Gutierrez et al. ${ }^{7)}$ analyzed factors by using a virtual model and found that varus humeral neck-shaft angle was the most important factor, followed by inferior positioning of the glenosphere, lateral offset of the glenosphere, and increased size of the glenosphere. In the current study, the ROC curve analysis indicated that inferior glenosphere overhang (AUC, 0.770) was the most important factor for reducing scapular notching, followed by increased PSNA (AUC, 0.727), greater glenoid neck length (AUC, 0.655), varus humeral neck-shaft angle (AUC, 0.625), and decreased IBPT (AUC, 0.564). In disagreement with previous studies, lateralization showed no significant effect on the incidence of scapular notching in this Korean population. The existing in vivo studies that reported decreased scapular notching with lateralization did not compare the incidence of notching between groups with medialized/lateralized COR, ${ }^{18,23)}$ and few clinical study included a variable of inferior glenosphere overhang. Furthermore, the anatomical difference between
Asian and western populations including the acromial length ${ }^{10)}$ and glenoid neck length probably contribute to this discrepancy. Further studies are necessary to confirm that better clinical outcomes can also be achieved by using inferior glenosphere overhang.

The present study has several limitations. First, given its retrospective design, unaccounted for factors or confounding bias such as the use of different types of implants and surgeries done by two surgeons may have distorted the results. Second, the mean follow-up period was relatively short (20.6 months); a longer follow-up is needed to evaluate long-term clinical outcomes and the occurrence of delayed complications, such as scapular notching progression, loosening of components, and acromial insufficiency. Lastly, this study only evaluated prognostic radiological factors; it did not conduct a comprehensive analysis of factors associated with characteristics of the patient (age, sex, preoperative deltoid status, preoperative range of motions, etc.) and surgeon (surgical technique, etc.).

More distalization and medialization of the COR during RSA would help reverse pseudoparalysis, and lateralization would improve active external rotation. Inferior glenosphere overhang was most important for preventing scapular notching and improving functional outcomes. Therefore, proper amount of deltoid lengthening (mean, $2.3 \mathrm{~cm}$ ) and inferior glenosphere overhang (mean, 2.9 $\mathrm{mm}$ ) should be chosen for the better outcomes, while positioning of the COR should be individualized based on patient characteristics in the Korean population.

\section{CONFLICT OF INTEREST}

No potential conflict of interest relevant to this article was reported.

\section{REFERENCES}

1. Boileau P, Watkinson D, Hatzidakis AM, Hovorka I. Neer Award 2005: the Grammont reverse shoulder prosthesis: results in cuff tear arthritis, fracture sequelae, and revision arthroplasty. J Shoulder Elbow Surg. 2006;15(5):527-40.

2. Guery J, Favard L, Sirveaux F, Oudet D, Mole D, Walch G. Reverse total shoulder arthroplasty: survivorship analysis of eighty replacements followed for five to ten years. J Bone Joint Surg Am. 2006;88(8):1742-7.

3. Ernstbrunner L, Suter A, Catanzaro S, Rahm S, Gerber C. Reverse total shoulder arthroplasty for massive, irreparable rotator cuff tears before the age of 60 years: long-term results. J Bone Joint Surg Am. 2017;99(20):1721-9.
4. Gerber C, Canonica S, Catanzaro S, Ernstbrunner L. Longitudinal observational study of reverse total shoulder arthroplasty for irreparable rotator cuff dysfunction: results after 15 years. J Shoulder Elbow Surg. 2018;27(5):831-8.

5. Li X, Knutson Z, Choi D, et al. Effects of glenosphere positioning on impingement-free internal and external rotation after reverse total shoulder arthroplasty. J Shoulder Elbow Surg. 2013;22(6):807-13.

6. Berhouet J, Garaud P, Favard L. Evaluation of the role of glenosphere design and humeral component retroversion in avoiding scapular notching during reverse shoulder arthroplasty. J Shoulder Elbow Surg. 2014;23(2):151-8. 
7. Gutierrez S, Levy JC, Frankle MA, et al. Evaluation of abduction range of motion and avoidance of inferior scapular impingement in a reverse shoulder model. J Shoulder Elbow Surg. 2008;17(4):608-15.

8. Levigne C, Boileau P, Favard L, et al. Scapular notching in reverse shoulder arthroplasty. J Shoulder Elbow Surg. 2008;17(6):925-35.

9. Hoenecke HR Jr, Flores-Hernandez C, D'Lima DD. Reverse total shoulder arthroplasty component center of rotation affects muscle function. J Shoulder Elbow Surg. 2014;23(8):1128-35.

10. Cabezas AF, Krebes K, Hussey MM, et al. Morphologic variability of the shoulder between the populations of North American and East Asian. Clin Orthop Surg. 2016;8(3):2807.

11. Matsuki K, Sugaya H, Hoshika S, et al. Geometric analysis of the proximal humerus in elderly Japanese patients: implications for implant selection in reverse shoulder arthroplasty. Orthopedics. 2017;40(3):e485-90.

12. Wall B, Nove-Josserand L, O'Connor DP, Edwards TB, Walch G. Reverse total shoulder arthroplasty: a review of results according to etiology. J Bone Joint Surg Am. 2007;89(7):1476-85.

13. Nyffeler RW, Werner CM, Gerber C. Biomechanical relevance of glenoid component positioning in the reverse Delta III total shoulder prosthesis. J Shoulder Elbow Surg. 2005;14(5):524-8.

14. Jobin CM, Brown GD, Bahu MJ, et al. Reverse total shoulder arthroplasty for cuff tear arthropathy: the clinical effect of deltoid lengthening and center of rotation medialization. J Shoulder Elbow Surg. 2012;21(10):1269-77.

15. Paisley KC, Kraeutler MJ, Lazarus MD, Ramsey ML, Williams GR, Smith MJ. Relationship of scapular neck length to scapular notching after reverse total shoulder arthroplasty by use of plain radiographs. J Shoulder Elbow Surg. 2014;23(6):882-7.

16. Simovitch RW, Zumstein MA, Lohri E, Helmy N, Gerber C. Predictors of scapular notching in patients managed with the Delta III reverse total shoulder replacement. J Bone Joint Surg Am. 2007;89(3):588-600.
17. Sirveaux F, Favard L, Oudet D, Huquet D, Walch G, Mole D. Grammont inverted total shoulder arthroplasty in the treatment of glenohumeral osteoarthritis with massive rupture of the cuff: results of a multicentre study of 80 shoulders. J Bone Joint Surg Br. 2004;86(3):388-95.

18. Frankle M, Siegal S, Pupello D, Saleem A, Mighell M, Vasey M. The reverse shoulder prosthesis for glenohumeral arthritis associated with severe rotator cuff deficiency: a minimum two-year follow-up study of sixty patients. J Bone Joint Surg Am. 2005;87(8):1697-705.

19. Ladermann A, Williams MD, Melis B, Hoffmeyer P, Walch G. Objective evaluation of lengthening in reverse shoulder arthroplasty. J Shoulder Elbow Surg. 2009;18(4):588-95.

20. Van Hoof T, Gomes GT, Audenaert E, Verstraete K, Kerckaert I, D'Herde K. 3D computerized model for measuring strain and displacement of the brachial plexus following placement of reverse shoulder prosthesis. Anat Rec (Hoboken). 2008;291(9):1173-85.

21. Gerber C, Pennington SD, Nyffeler RW. Reverse total shoulder arthroplasty. J Am Acad Orthop Surg. 2009;17(5):28495.

22. Roche CP, Stroud NJ, Flurin PH, Wright TW, Zuckerman JD, DiPaola MJ. Reverse shoulder glenoid baseplate fixation: a comparison of flat-back versus curved-back designs and oval versus circular designs with 2 different offset glenospheres. J Shoulder Elbow Surg. 2014;23(9):1388-94.

23. Cuff D, Pupello D, Virani N, Levy J, Frankle M. Reverse shoulder arthroplasty for the treatment of rotator cuff deficiency. J Bone Joint Surg Am. 2008;90(6):1244-51.

24. Walker M, Brooks J, Willis M, Frankle M. How reverse shoulder arthroplasty works. Clin Orthop Relat Res. 2011;469(9):2440-51.

25. Boulahia A, Edwards TB, Walch G, Baratta RV. Early results of a reverse design prosthesis in the treatment of arthritis of the shoulder in elderly patients with a large rotator cuff tear. Orthopedics. 2002;25(2):129-33.

26. Boileau P, Watkinson DJ, Hatzidakis AM, Balg F. Grammont reverse prosthesis: design, rationale, and biomechanics. J Shoulder Elbow Surg. 2005;14(1 Suppl S):147S-161S. 